Available online on 15.11.2016 at http://jddtonline.info
(c) 2016, publisher and licensee JDDT, This is an Open Access article which permits unrestricted
noncommercial use, provided the original work is properly cited

Research Article

\title{
EVALUATION OF THE PRESCRIBING PRACTICE BASED ON WORLD HEALTH ORGANIZATION CORE PRESCRIBING INDICATORS IN HIWOT FANA SPECIALIZED UNIVERSITY HOSPITAL, HARAR, EASTERN ETHIOPIA
}

\author{
Tigist Getachew $^{1}$, Yohannes Tadiwos ${ }^{2}$, Mekonnen Sisay ${ }^{2 *}$ \\ ${ }^{1}$ Karamara General Hospital, Jigjiga, eastern Ethiopia \\ ${ }^{2}$ Department of Pharmacology and Toxicology, School of Pharmacy, College of Health and Medical Sciences, Haramaya University, Harar, \\ eastern Ethiopia
}

\begin{abstract}
Background: Drugs are an important component of health care and play a crucial role in saving life. Drug use in a health facility is a complex subject involving physician, patient and dispenser and hence is directly affected by prescribing, dispensing and patient use of drugs. The study is, therefore, aimed to evaluate drug prescribing practice using World Health Organization (WHO) prescribing indicators in Hiwot Fana Specialized University Hospital (HFSUH), Harar, Ethiopia.

Method: A retrospective cross sectional study was carried out to determine the current prescribing practice in HFSUH outpatient pharmacy department, Harar, from January 20-February 20, 2016. As per the WHO guidelines, 600 prescription papers were selected using systematic random sampling and reviewed with the help of observational checklist. The data were entered and processed with SPSS version 16. Finally, data were evaluated using WHO criteria.

Result: The average number of drugs prescribed per encounter was 1.7. The percentage of encounters in which an antibiotic or injection was prescribed was 57.2\% (343) and 59\% (354), respectively. Besides, the percentage of drugs prescribed by generic name and from an essential drug list (EDL) was found to be $92.1 \%$ (954) and $68.94 \%$ (708), respectively.

Conclusion: On the basis of these findings, the prescribing practices for antibiotic use; injection use; and prescribing from EDL showed a great deviation from the standards recommended by WHO. These two commonly overused and costly forms of drug therapy (antibiotics and injections) need to be regulated closely. In addition, prescribing practice should be limited to EDL of the country.
\end{abstract}

Key words: WHO, core prescribing indicators, prescribing practice, HFSUH

Article Info

Received 14 Oct 2016; Review Completed 06 Nov 2016; Accepted 10 Nov 2016, Available online 15 Nov 2016

Article URI: http://jddtonline.info/index.php/jddt/article/view/1344

Cite this article as:

Getachew T, Tadiwos Y, Sisay M, Evaluation of the prescribing practice based on world health organization core prescribing indicators in Hiwot Fana Specialized University Hospital, Harar, Eastern Ethiopia, Journal of Drug Delivery \& Therapeutics. 2016; 6(6):25-30 DOI: http://dx.doi.org/10.22270/jddt.v6i6.1344

*Corresponding author:

Mekonnen Sisay (BPharm, MSc in Pharmacology), Department of Pharmacology and Toxicology, School of Pharmacy, College of Health and Medical Sciences, Haramaya University, Harar, P.O.Box 235, Ethiopia,

Mail ID: mekonnensisay27@yahoo.com Cell phone: +251920-21-21-35

Abbreviations

EDL: Essential drug list

RDU: Rational Drug Use
HFSUH: Hiwot Fana Specialized University Hospital INRUD: International Network for Rational Use of Drugs
STG: Standard Treatment Guideline WHO: World Health Organization 


\section{INTRODUCTION}

The rational use of medications is a worldwide concern, and is prominent among health care professionals. Concerted efforts to readjust pharmaceutical actions and practices aiming at the rational use of medication are essential in today's society, in which drugs are the most used therapeutic resources. Aiming to evaluate the conditions of the services offered to the population concerning medication. WHO developed core medication use indicators which are prescribing indicators, patient care indicators and health facility indicators. $^{1,2}$

Essential drugs comprise one of the tools needed to fight ill health. By increasing access to essential drugs and their rational use, we could improve health status and secure development gains. "Essential drugs are those that satisfy the health care needs of the majority of the population; they should therefore be available at all times; in adequate amounts and in the appropriate dosage forms". 4 This concept was introduced to accelerate the positive impacts of drugs on health status, particularly for developing countries. ${ }^{3}$

Irrational prescribing is a global problem. Bad prescribing habits lead to ineffective and unsafe treatment, exacerbation or prolongation of illness, distress and harm to the patient, and higher costs. Irrational prescribing patterns are perpetuated through patient pressure, bad example of colleagues, and highpowered salesmanship by drug company representatives. In teaching hospitals, new graduates will copy them, completing the vicious circle. Changing existing prescribing habits becomes very difficult. ${ }^{5}$

The overuse, underuse or misuse of medicines results in wastage of scarce resources and widespread health hazards. Examples of irrational use of medicines include use of too many medicines per patient ("polypharmacy"); inappropriate use of antimicrobials, often inadequate dosage; over-use of injections when oral formulations would be more appropriate; failure to prescribe in accordance with clinical guidelines; nonadherence to dosing regimens. ${ }^{6}$

Antimicrobial resistance is an increasing problem worldwide, impacting infection control efforts and costs of antimicrobial treatment. Ethiopian hospitals consume about $50 \%$ of the national drug budget, which is considered to be high drug budget compared to the population segment using these health facilities. However, very little is known how drugs (particularly antibiotics) are used in hospitals, like in other health facilities. $^{7}$ Previous studies have showed that inappropriate use of antibiotics is central to the development and spread of resistant bacteria in hospitals and community. ${ }^{8}$

Assessment of drug use patterns with the WHO drug use indicators is becoming increasingly necessary to promote rational drug use in developing countries, before activities are started to promote rational drug use, an effort should be made to describe and quantify the situation. Several well-established survey methods are available for this purpose. One assessment method is a prescribing and patient care survey using the WHO drug use indicators. These quantitative indicators are now widely accepted as a global standard for problem identification and have been used in over 30 developing countries. ${ }^{9}$ Therefore, this study was aimed to evaluate the existing prescribing practices using WHO core prescribing indicators at HFSUH outpatient pharmacy departments during the study period.

\section{METHODS}

\section{Study area, design and period}

The study was conducted in HFSUH outpatient pharmacy department, Harar which is located $526 \mathrm{~km}$ from Addis Ababa, capital of Ethiopia. A retrospective cross sectional study design was carried out from January 20 to February 20, 2016.

\section{Population}

All prescriptions dispensed at HFSUH outpatient pharmacy from September to December, 2015 were taken. Prescriptions containing only medical supplies, prescriptions for immunization, for pregnancy management, for malaria and for prophylaxis therapy were excluded from the study. In addition, prescription papers with insufficient information were also excluded.

\section{Sample size and Sampling technique}

According to the WHO/INRUD recommendation, a total of at least 600 prescription papers are required for the assessment of drug prescribing indicators among health facilities. ${ }^{1}$ In this study, a total of 600 prescription papers from the outpatient pharmacy of HFSUH were included to enhance the validity and reliability of the data.

The aforementioned sample size of prescribing encounters was taken by systematic random sampling from OPD pharmacy of HFSUH. Afterwards, each prescription paper was reviewed retrospectively.

$$
\mathrm{K}=\frac{\text { Total number of prescriptions }}{600}=\frac{7560}{600}=12.6 \approx 13
$$

Where, K=interval of sampling, just after the first prescription is randomly selected

\section{Data collection procedures}

Data were collected by using WHO designed criteria based data collection formats (observational check lists) for retrospective study. According to WHO drug use evaluation guideline, outpatient prescribing indicators includes- the average number of drugs per encounter, percentage of drugs prescribed in generics, percentage of prescriptions with antibiotics, percentage of prescriptions with injections and percentage of prescribed drugs from EDL. These specific types of data that were necessary to measure the prescribing indicators were recorded and entered directly into an ordinary prescribing indicator form. 


\section{Variables}

\section{Independent variables}

* Average number of drugs per encounter.

* Percentage of encounter with an antibiotic prescribed.

* Percentage encounter with an injection prescribed.

* Percentage of drugs prescribed by generic name.

* Percentage of drugs prescribed from EDL or formulary.

\section{Dependent variables}

\section{Prescribing practice}

\section{Data quality control}

After collecting and sorting the data, all records were checked for errors. The data were cleared every day and prescription papers with insufficient information were excluded from the study. Then the data were converted into quantifiable numerical form in order to analyze it with the statistical package for social science (SPSS16.1).

\section{Data processing and analysis}

Abstracted information from the prescription sheets was entered into the data collection form and sorted appropriately and then the necessary data on prescribing indicators were calculated and summarized on the appropriate form. After the data had been collected from the prescriptions, the results were entered. Afterwards, it was analyzed by SPSS. Prescribing indicators were then calculated, using the WHO guideline. ${ }^{1}$

\section{Ethical consideration}

Approval and permission was sought from Institutional Research Ethics Review Committee, College of Health and Medical Sciences, Haramaya University. Besides, permission paper written by School of Pharmacy, Haramaya University was submitted to HFSUH to undertake the study

\section{Limitation of the study}

The prescribing indicators measure aspects of outpatient treatment. They are designed for use in health centers, dispensaries or hospital outpatient departments. The prescribing indicators are less useful in specialty outpatient clinics in referral hospitals, where the drug use pattern is more complex. WHO prescribing indicators only record what is prescribed and does not address the reason why the problem exists.

\section{RESULTS}

A total of 600 prescription papers were collected and analyzed using WHO guidelines. Out of 600 prescriptions that were collected and analyzed, the total number of drugs prescribed was found to be 1027. Most of the prescriptions contain one drug $51.8 \%$ (311) followed by two drugs $32.3 \%$ (194), and three drugs $10.7 \%$ (64) (Figure 1)

The average number of drugs prescribed per encounter was 1.71. Percentage of prescription with an antibiotic prescribed was $57.2 \%$ (343) and percentage of encounters with at least one injection was 59\% (354). The total percentage of drugs prescribed with generic name was found to be $92.89 \%$ (954) and percentage of drug prescribed from EDL was 68.94\% (708) (Table 1).

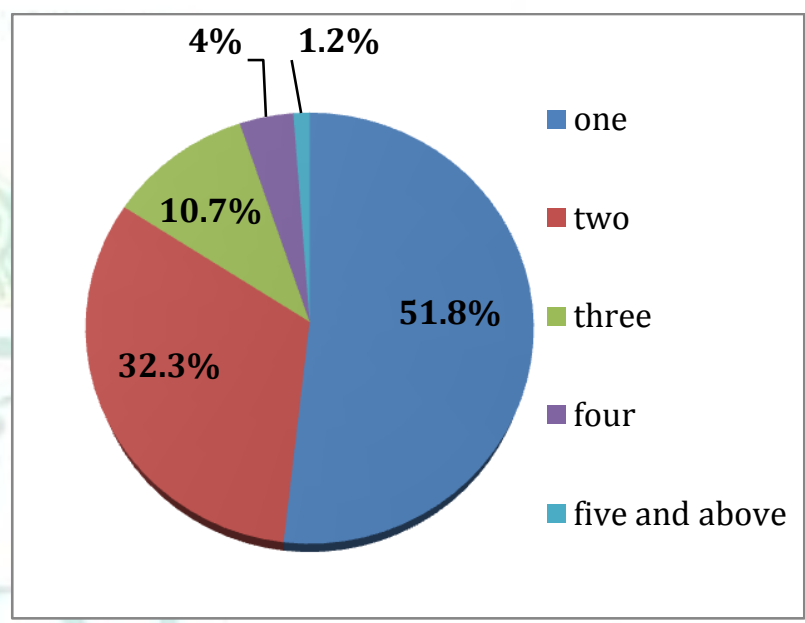

Figure 1: percentage distribution of prescriptions with number of drugs per encounters at HFSUH, OPD from Sep to Dec, $2015(n=1027)$

Table 1: Summary of five core prescribing indicators obtained at HFSUH outpatient prescriptions dispensed from Sep to Dec, $2015(\mathrm{n}=600)$

\begin{tabular}{|l|l|l|}
\hline Prescribing indicators & HFSUH & Standard (WHO) \\
\hline $\begin{array}{l}\text { Total drugs prescribed } \\
\text { Total number of prescriptions studied }\end{array}$ & 1027 & \\
\hline Average number of drugs per Encounter & 600 & $1.6-1.8$ (ideal) (<2) \\
\hline Percentage of drugs prescribed by generics & 1.7 & $100 \%$ \\
\hline Percentage of encounter with antibiotics & $954(92.9 \%)$ & $20.0-26.8 \%-24.1 \%$ \\
\hline Percentage of encounters with injection & $343(57.2 \%)$ & $13.4-24.1 \%$ \\
\hline Percentage of drugs from essential drug list & $354(59 \%)$ & $100 \%$ \\
\hline
\end{tabular}

*HFSUH: Hiwot Fana Specialized University Hospital; WHO: World health organization

Coming to the number of drugs prescribed from EDL of Ethiopia, most of the prescriptions contain one drugs prescribed from EDL (51.7\%) followed by none of the drugs prescribed from EDL (19.8\%) and two of the drugs prescribed from EDL (19.7\%) (Figure 2). 


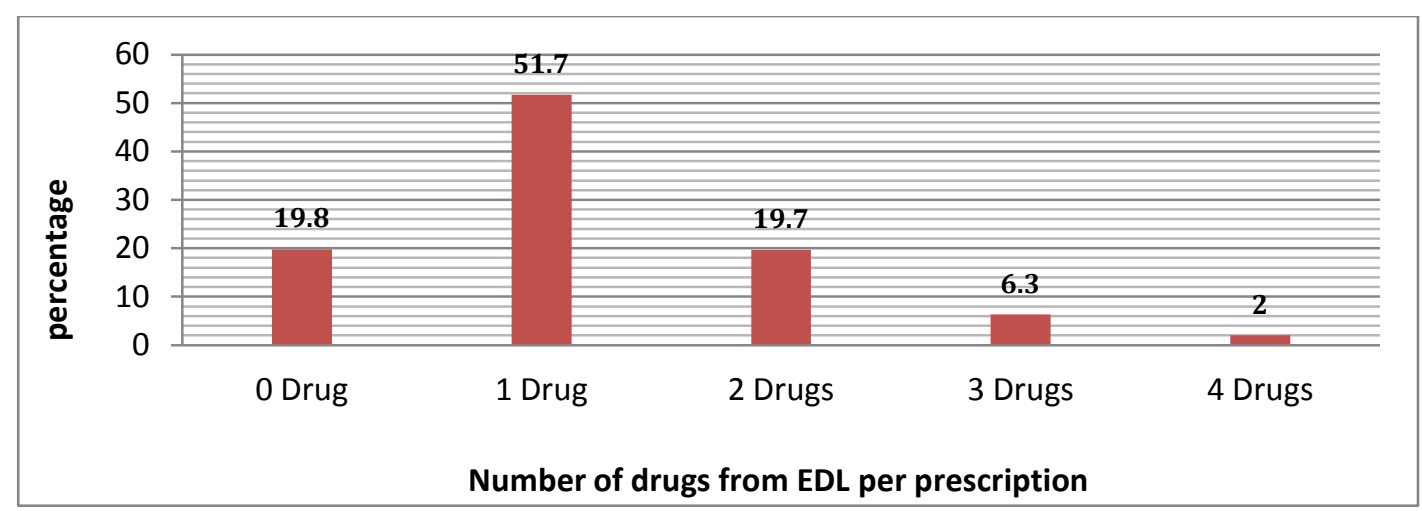

Figure 2: The percentage distribution of the number of drugs prescribed from the essential drug lists per prescription at HFSUH outpatient pharmacy from Sep to Dec, 2015 GC (n=1027).

Concerning the generic prescribing practice in the hospital, almost half of prescribing encounters contain one drug prescribed with generic name $(52.7 \%)$ followed by two drugs (30.3\%) and three drugs (11\%) prescribed with generics (Figure 3).

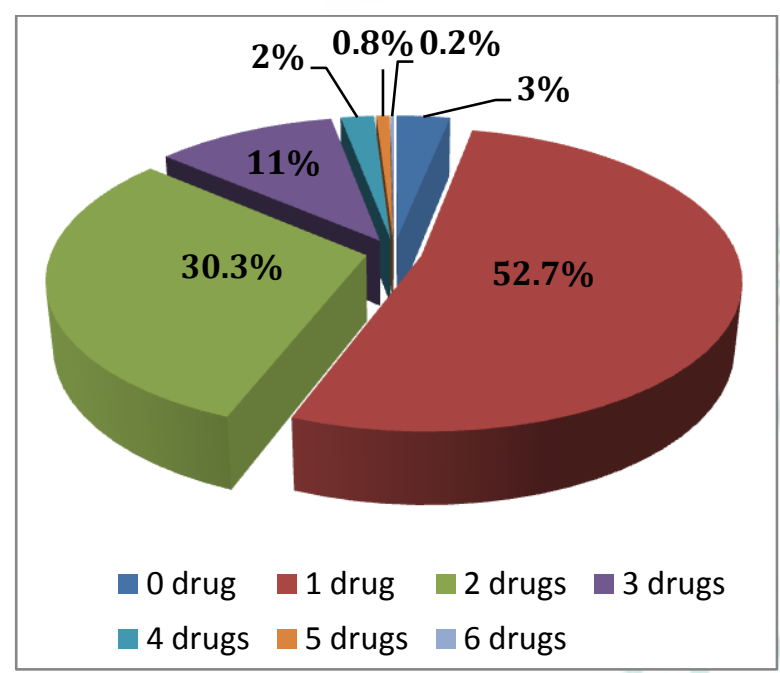

Figure 3: Percentage distribution of drugs prescribed by generic name per prescription at HFSUH outpatient pharmacy from Sep to Dec, 2015 GC $(\mathbf{n}=1027)$

\section{DISCUSSION}

WHO developed a core prescribing indicators to measure the degree of poly pharmacy, the tendency to prescribe drugs by generic name and the overall level of use of antibiotics and injections. The degree to which the prescribing practice is in accordance with the EDL, formulary or standard treatment guideline was also measured by searching the number of drugs prescribed from the EDL. ${ }^{10}$ Accordingly, the result of this study revealed that the average number of drugs per encounter was 1.71 , which is in line with the WHO standard value, that is less than or equal to two. ${ }^{1,11}$ This shows that there had been proper use of drugs per prescription at HFSUH.

The average number of drugs per prescription (1.7) for HFSUH is acceptable compared with the standard (1.61.8) derived as ideal. In a similar study performed in southern Ethiopia at Hawassa university teaching and referral hospital (HUTRH), the average number of drugs per encounter was found to be $1.9 .^{12}$ In another study in Northern Ethiopia at Ayder referral hospital (ARH), the average number of drugs per prescription was $2.61 .^{13}$ This value is higher than the value recommended by WHO as a standard and which is also higher than the findings of this study. However, in a study on prescribing patterns in three hospitals in North Ethiopia, the average number of drugs per patient was 0.98 at Gondar Hospital, 1.8 in Bahirdar Hospital, and 2.2 in Debre Tabor Hospital. ${ }^{14}$ A national baseline study on drug use indicators in Ethiopia in September 2002 also found the average number of drugs prescribed per encounter to be 1.9, which is comparable to our finding. ${ }^{15}$

Regarding the generic prescribing practice, the percentage of drugs prescribed by generic name was $92.89 \%$, which is somewhat lower compared to the standard derived to serve as ideal $(100 \%) .{ }^{1}$ However, lower values of generic prescribing practices were reported in several health care settings such as selected hospitals of west Ethiopia $(79.2 \%)^{16}$, Nigerian army hospitals $(49.3 \%){ }^{17}$, secondary care referral hospital of south India $(42.9 \%)^{18}$, and Jimma Hospital, south-west Ethiopia $(75.2 \%)^{19}$. In a similar study carried out at HUTRH, southern Ethiopia, the percentage of drugs prescribed by generic name was $98.7 \%$, which is almost similar compared to the standard but higher compared to this finding ${ }^{12}$. A study on evaluation of drug prescribing pattern using WHO prescribing indicators in ARH, northern Ethiopia, showed the percentage of drugs prescribed by generic name to be $93.3 \%$, which is similar to our finding of $92.89 \%{ }^{13}$. Prescribing by generic name is recommended by WHO as it enables selection of more alternatives and it has also a major impact in cost minimization. The factor influencing low generic prescribing may be due to poor regulation and enforcement, drug promotion and less number of generic drug productions in Ethiopia. A national baseline study on drug use indicators in Ethiopia in September 2002 also showed the percentage of drugs prescribed by generic name to be $87 \%$, which is lower than our finding of $92.89 \%$. $^{15}$

Regarding antibiotics prescribing, there was unacceptably high use of antibiotics (57.2\%). The value 
from this study was less than the value obtained from a study conducted at Debretabor Hospital in Ethiopia $69 \% .^{14}$, comparable to a study carried out at HUTRH, south Ethiopia $58.1 \%^{12}$ and high compared to a study in $\mathrm{ARH}$, northern Ethiopia which is $32 \%{ }^{13}$ WHO recommends that less than $30 \%$ of antibiotic encounters is expectable in the countries where an infectious disease is more prevalent. Overuse of antibiotics in HFSUH could play a significant role in increasing antimicrobial resistance to currently available costeffective antibiotics. Therefore, prompt measures should be taken to improve the prescribing habits and the use of these types of drugs. This finding suggests that antibiotic prescribing needs to be regulated. The high percentage of antibiotics prescribed in our study setting may be due to patient expectation to receive antibiotics, or prescribers' belief that the therapeutic efficacy of antibiotics is low. A national baseline study on drug use indicators in Ethiopia in September 2002 also showed that the percentage of encounters in which an antibiotic was prescribed to be $58.1 \%$, which was similar to our finding. ${ }^{15}$

WHO recommended that less than $10 \%$ prescription should include one or more injections. ${ }^{1}$ The result of this study revealed that percentage of injection prescribed was $59 \%$, which is very high compared to a study conducted in HUTRH ${ }^{12}$, ARH $23.6 \%{ }^{13}$, Indian health care setting ${ }^{20}$ Zimbabwe ${ }^{21}$, Southern Ethiopia ${ }^{22}$, Gondar university hospital ${ }^{23}$ and HUTRH ${ }^{12}$, and west Ethiopia. $^{16}$

This shows that the use of injection in this hospital was very high and this could lead to variety of problem that are associated with injection treatments like sepsis at administration, the risk of tissue toxicity from local irritation, increased cost of treatment and, difficulties in correcting the error. ${ }^{1,11}$

The percentage of drug prescribed from EDL was less compared to the standard $(100 \%)$ derived to serve as the ideal. According to the result of this study, $68.94 \%$ were prescribed from EDL, which is less when compared to other study in HUTRH $(96.6 \%)^{12}$ and ARH $100 \% .^{13}$

\section{CONCLUSION}

On the basis of the findings, the prescribing practices for antibiotic use, prescribing from EDL and injection use showed a great deviation from the standards recommended by WHO. Overuse of antibiotics $(57.2 \%)$ is a problem because it could facilitate the emergence of antimicrobial resistance which is a major bottleneck for our globe. Generic prescribing showed little deviation from the standard whereas the average number of drug per encounter lay within the range of WHO. Non generic prescribing may result in high treatment cost and could create a problem of adherence.

\section{ACKNOWLEDGEMENTS}

We would like to thank Haramaya University for provision of material supports. We also extend our deepest gratitude and appreciation for HFSUH staffs for their kindly support.

\section{COMPETING INTERESTS:}

The authors have declared that there is no competing interest

\section{AUTHORS' CONTRIBUTION}

TG was involved in the conception of the original idea, helped to draft the proposal, participated in all implementation stages of the project, and write up; YT and MS supervised the research; MS also prepared the final manuscript for publication. All authors read and approved the final version of the manuscript.

Funding: No specific grant received for this study

\section{REFERENCES}

1. WHO, how to investigate drug use in health facilities: selected drug indicators, action program on essential drugs (DAP), Geneva, 1995.

(http://apps.who.int/medicinedocs/en/d/JS/2289e/1.1.html\#JS 2289e.1.1)

2. Vooss AT, Diefenthaeler HS. Evaluation of prescription indicators established by the WHO in Getúlio Vargas-RS. Braz J Pharm Sci. 2011;47 (2): 385-90.

3. WHO. Medicines Strategy: Framework for action Essential drugs and Medicines policy 2000-2003. Geneva (WHO/EDM/2000.1).

4. WHO (1995). The use of Essential Drugs. 6th Report of the Expert Committee. Geneva. WHO Technical Report Series 850.3. Available at

whqlibdoc.who.int/trs/WHOTRS_850.pdf

5. FMHACA of Ethiopia. Manual for Medicines Good Prescribing Practice: $2^{\text {nd }}$ edition. Addis Ababa. 2012. Available at www.fmhaca.gov.et/documents/Medcines

6. Sosola AG. An assessment of prescribing and dispensing practices in public health facilities of Southern Malawi, Malawi University. 2007 available at apps.who.int/medicinedocs/documents/s21439en/s21439en.p df

7. Ministry of Health. Master plan for the Ethiopian national drugs programme, Addis Ababa.1994

8. DACA (2009). Antimicrobials use, resistance and containment baseline survey syntheses of findings, Addis Ababa, Ethiopia.

9. Laing RO, Hogerzeil HV, Ross-Degnan D. Ten recommendations to improve use of medicines in developing countries. Health policy and planning. 2001; 16(1):13-20.

10. WHO. Promoting Rational Use of Medicines: Core Components - WHO Policy Perspectives on Medicines, No. 005, September 2002 at http://apps.who.int/medicinedocs/en/d/Jh3011e/

11. Mallet HP, Njikam A, Scouflaire SM. Evaluation of prescription practices and of the rational use of medicines in Niger. Sante (Montrouge, France). 2000; 11(3):185-93.

12. Desalegn AA. Assessment of drug use pattern using WHO prescribing indicators at Hawassa University teaching and referral hospital, south Ethiopia: a cross-sectional study. BMC health services research. 2013; 13(1):1.

13. Berhanu D, Molla F, Assen A, Melkam W, Abrha S, Masresha B, et al. Evaluation of drugs utilization pattern using WHO prescribing indicators in Ayder referral hospital, Northern Ethiopia. IJPSR. 2015; 6 (2): 343-347.

14. Desta Z, Abula T, Gebre-Yohannes A, Worku A. Prescribing patter for outpatients in three hospitals in north-west Ethiopia. J. Health Dev. 2002; 16(2): 183-189.

15. WHO and MOH. Assessment of the Pharmaceutical Sector in Ethiopia. Addis Ababa.2003. Available at http://www. who.int/medicines/areas/coordination/ethiopia_ pharmaceutical.pdf.

16. Lenjisa JL, Fereja TH. A Retrospective Analysis of Prescribing Practice Based on WHO Prescribing Indicators at 
Four Selected Hospitals of West Ethiopia: Policy Implication. East and Central African Journal of Pharmaceutical Sciences. 2013; 16(3):69-74.

17. Adebayo ET and Hussain NA. Pattern of prescription drug use in Nigerian army hospitals. Annals of African Medicine. 2010; 9 (3):152-8

18. Prasad PS, Rudra JT, P Vasanthi, Sushitha U. Assessment of drug use pattern using World Health Organization core drug use indicators at Secondary Care Referral Hospital of South India. CHRISMED Journal of Health and Research 2015; 2(3): $223-8$

19. Abdulahi M, Shiferaw T. Pattern of prescription in Jimma Hosptial. Ethiop J Health Dev.1997; 11(3): 263-267.

20. Reddy PV., Ravichandran V and Shanmugasundaram P. evaluation of drug use pattern in gynecology department using WHO prescribing indicators, Int J Pharm Bio Sci 2015; 6(4): $83-88$

21. Ministry of Health/Zimbabwe Essential Drugs Action Programme: Essential Drugs Survey. In How to investigate drug use in health facilities. WHO:Geneva; 1993. 74(WHO/DAP/93.1)

22. Dilbato DD, Kuma ZG, and T/Mariam S. A base line survey on prescribing indicators and the underlying factors influencing prescribing in southern Ethiopia. Ethiopian Journal Health Development. 1998; 12(2): 87-93.

23. Admassie E, Begashaw B and Hailu W. Assessment of drug use practices and completeness of prescriptions in Gondar University Teaching Referral Hospital. International journal of pharmaceutical sciences and research. 2013; 4 (1): 265 275 . 